\title{
DSCC2010-व००
}

\section{MATHEMATICAL MODELLING OF NEAR-HOVER INSECT FLIGHT DYNAMICS}

\author{
B. Cheng \\ $X$. Deng \\ School of Mechanical Engineering, Purdue \\ University, West Lafayette, IN 47907 \\ xdeng@purdue.edu
}

\begin{abstract}
Using a dynamically scaled robotic wing, we studied the aerodynamic torque generation of flapping wings during roll, pitch, and yaw rotations of the stroke plane. The total torque generated by a wing pair with symmetrical motions was previously known as flapping counter-torques (FCTs). For all three types of rotation, stroke-averaged FCTs act opposite to the directions of rotation and are collinear with the rotational axes. Experimental results indicate that the magnitude of FCTs is linearly dependent on both the flapping frequency and the angular velocity. We also compared the results with predictions by a mathematical model based on quasi-steady analyses, where we show that FCTs can be described through consideration of the asymmetries of wing velocity and the effective angle of attack caused by each type of rotation. For roll and yaw rotations, our model provided close estimations of the measured values. However, for pitch rotation the model tends to underestimate the magnitude of FCT, which might result from the effect of the neglected aerodynamics, especially the wake capture.
\end{abstract}

Similar to the FCT, which is induced by body rotation, we further provide a mathematical model for the counter force induced by body translation, which is termed as flapping counter-force (FCF). Based on the FCT and FCF models, we are able to provide analytical estimations of stability derivatives and to study the flight dynamics at hovering. Using fruit fly (Drosophila) morphological data, we calculated the system matrix of the linearized flight dynamics. Similar to previous studies, the longitudinal dynamics consist of two stable subsidence modes with fast and slow time constants, as well as an unstable oscillatory mode. The longitudinal instability is mainly caused by the FCF induced by an initial forward/backward velocity, which imparts a pitch torque to the same direction of initial pitch velocity. Similarly, the lateral dynamics also consist of two stable subsidence modes and an unstable oscillatory mode. The lateral instability is mainly caused by the FCF induced by an initial lateral velocity, which imparts a roll torque to the same direction of initial roll velocity. In summary, our models provide the first analytical approximation of the six-degree-of-freedom flight dynamics, which is important in both studying the control strategies of the flying insects and designing the controller of the future flapping-wing micro air vehicles (MAVS).

\section{INTRODUCTION}

Recent studies on the tuning dynamics of animal flight [1, 2] showed that during low-speed yaw turns (rotation about the vertical axis such as saccade), flapping wing fliers ranging in size from fruit flies to large birds are subject to substantial passive damping through an aerodynamic mechanism termed flapping counter-torque (FCT). As an inherent property, FCT helps the flapping-wing fliers to slow down body rotation during rapid maneuvers and thus reduces the required active torque produced by asymmetries of wing motion. As a tradeoff, however, flapping-wing fliers must overcome extensive aerodynamic damping (a result of FCT) to accelerate or to initiate a maneuver [1]. Not only was the passive damping found crucial during fast yaw rotations, simulation results [1] suggested that it is also present during roll and pitch maneuvers. In flying animals, measurements of body kinematics showed that most yaw turns are accompanied by substantial change in roll angular velocity [3]. Even at low speed maneuvering or hovering, most flapping wing flies perform banked turns which involve rolling. Furthermore, during escape or tracking flight, rapid reorientations of roll and pitch angles (causing reorientation of the net aerodynamic force vector) are essential for fliers to achieve fast maneuvers $[4,5]$. 
Therefore, aerodynamic mechanisms that can achieve passive stabilization of roll and pitch angle is important in flapping flight.

Similar to the aerodynamic damping (from FCT) caused by body rotation, the aerodynamic damping caused by body translations may also be present and important to flight dynamics. It is clear that relative airspeed will induce a drag opposite to the flight direction and varying linearly with airspeed [6]. Previous work has also shown that the aerodynamics during forward flight is different from that during hovering [7]. Furthermore, it was shown that the turning rate of a saccade is inversely related to the forward velocity, which might reflect an aerodynamic constraint on the flight dynamics [8]. Therefore, a comprehensive understanding of aerodynamic damping, in all rotational and translational degrees of freedom, is crucial to the understanding of the flight dynamics of flapping flight and in the design of bio-inspired micro aerial vehicles.

Insect flight was shown to be inherently unstable based on simulations with computational fluid dynamics methods [9] and experiments with tethered locusts [10], and active modulation of wing kinematics is the key to achieving the observed maneuverability and stability. Our previous work on hovering dynamic models assumed negligible body velocities and therefore resulted in an open-loop marginally stable system [11]. However, it is evident that body velocities play an essential role in the aerodynamics and structural dynamics of flapping flight, and a comprehensive analytical modeling of insect flight dynamics is important to analyze flight stability and control.

In this study, we use a dynamically scaled robotic wing to investigate the aerodynamic damping (as a result of FCT) under roll, pitch, and yaw rotations of the stroke plane. We further present mathematical models for each type of rotation and compare their predictions with experimental results. We also provide mathematical models for aerodynamic damping during body translations, the mechanism of which we describe as flapping counter-force (FCF). The flight dynamics in nearhover conditions is investigated using FCT and FCF models to calculate the stability derivatives. In sum, we provide a comprehensive analytical approximation of the six-degree-offreedom flight dynamics.

\section{MATERIAL AND METHODS}

\section{Experimental setup}

The coordinate systems and wing kinematic parameters are described in Fig. 1A. The wings are fashioned from plastic sheet PET-G (thickness of 0.06 inch) with a length of $21 \mathrm{~cm}$ and an aspect ratio of 8.5 (including the lengths of the gearbox, sensor, and wing holder). The instantaneous force and torque acting on the wing were measured with a six-component forcetorque sensor (ATI NANO-18, Apex, NC, USA) attached to the wing holder (Fig. 1A). The wing and the gearbox $(2.54 \mathrm{~cm} \times$ $2.54 \mathrm{~cm} \times 2.54 \mathrm{~cm})$ were immersed in a tank $(46 \mathrm{~cm}$ wide $\times 41$ $\mathrm{cm}$ high $\times 152 \mathrm{~cm}$ long) filled with mineral oil (kinematic viscosity $=20 \mathrm{cSt}$ at $20^{\circ} \mathrm{C}$, density $=850 \mathrm{~kg} / \mathrm{m} 3$ ).

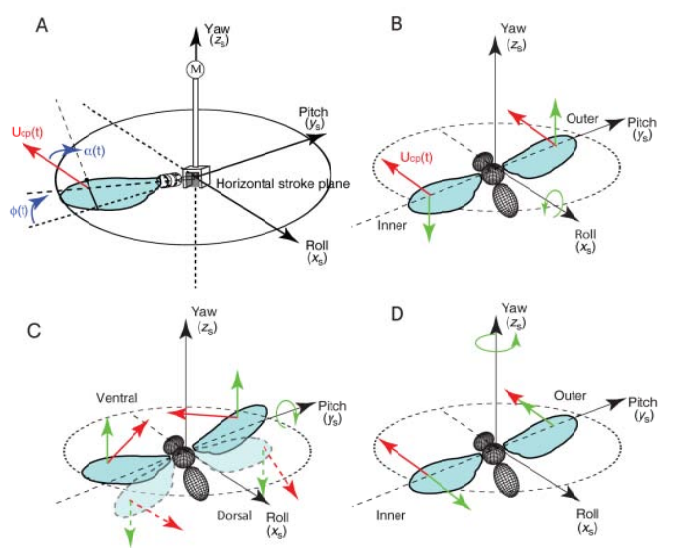

Fig. 1. Schematic view of wing kinematic parameters and rotations of the stroke plane. (A) A dynamically scaled robotic wing with the stroke plane frame. Wing kinematics are specified by the instantaneous stroke angle $\phi(t)$, and the rotation angle $\psi(t)$ (not shown here). The instantaneous angle of attack is defined as the angle between the wing chord and wing velocity $U_{\mathrm{cp}}(t)$. The kinematics of the left and right wings are played individually on the apparatus. (B) - (D) The wing kinematic asymmetries during the rotations about: (B) roll axis, (C) pitch axis, and (D) yaw axis. The green arrows represent the components of wing velocity induced by rotation of the stroke plane, and the red arrows represent the components of wing velocity relative to the stroke plane (under no rotation).

\section{Stroke kinematics and experimental procedures}

To see the effect of the stroke plane's rotation on the torque production of a wing pair (or to measure the FCT), we first superimposed the angular velocity of the stroke plane into the originally symmetrical wing motions (Fig. 2A). The resultant kinematics was then played through the dynamically scaled robotic wing. We performed the experiments with a single robotic wing because wing-wing interactions are negligible for most insects during hover $[12,13]$. Based on measured torques (in a coordinate frame attached to the sensor), the torques of a wing pair around three principal axes in the stroke-plane frame were calculated. The gravitational and inertial torques of the wing were measured by playing the wing kinematics in the air and subtracted the measurements from the total torque measured in the oil.

In all of the three independent sets of experiments, we rotated the stroke plane about each of its principal axes (roll $x_{\mathrm{s}}$, pitch $y_{\mathrm{s}}$, and yaw $z_{\mathrm{s}}$, Fig. 1A). For roll and pitch rotations, the wing strokes started with a tilted-stroke plane, passing the horizontal plane, and stopping at another tilted-stroke plane (Fig. 2B and C). For yaw rotation, the stroke plane was always horizontal (Fig. 2D). Note that the wing was started impulsively from rest, and to establish similar initial conditions during continuous strokes, we played two symmetrical wing strokes in advance and started the rotation at the third stroke.

In each set of experiments we systematically varied the flapping frequency $(n)$ and angular velocity $(\omega)$ to investigate their effects on the torque production. We first fixed the angular 
velocity at $8^{\circ} \mathrm{s}^{-1}$ and varied flapping frequency from $0.15 \mathrm{~Hz}$ to $0.45 \mathrm{~Hz}$ with 0.05 increments. The Reynolds number associated with this range of flapping frequency was from 300 to 900 , which is typical for insect flight [14]. We then fixed the flapping frequency at $0.4 \mathrm{~Hz}(\mathrm{Re}=800)$ and varied the angular velocity from $2^{\circ} \mathrm{s}^{-1}\left(5^{\circ} / \mathrm{stroke}\right)$ to $14^{\circ} \mathrm{s}^{-1}\left(35^{\circ} /\right.$ stroke $)$ with $2^{\circ} \mathrm{s}^{-1}$ $\left(5^{\circ} /\right.$ stroke $)$ increments.

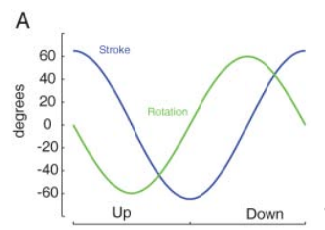

B Upstroke $\rightarrow$

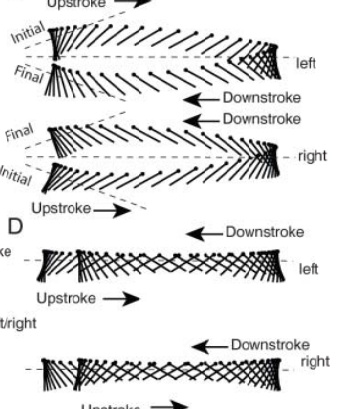

Fig. 2. Wing kinematics in the robotic wing experiments. (A) Plots of stroke angle $\phi$ (blue) and rotation angle $\varphi$ (green) varying as sinusoids $65 \cos \omega t$ and $60 \sin \omega t$, respectively. (B-D) Trajectories of the resultant wing kinematics resulting from rotations about the roll (B), pitch (C), and yaw (D) axes of the stroke plane. The solid black line denotes the wing chord, with the filled cycle marking the leading edge. The dashed black line indicates the orientation of the stroke plane.

\section{Theoretical estimations of flapping counter-torques}

The mechanism of FCT can be explained by considering the geometric asymmetries of wing kinematics caused by the stroke plane's rotation. Based on a quasi-steady aerodynamic analysis, we derived a mathematical model of FCT for each type of rotation. For yaw rotation, the FCT arises from the asymmetries of (wing) velocity magnitude between the inner and outer wings (Fig. 1D) [1,2]. The instantaneous FCT at nondimensional time $\hat{t}(\hat{t}=t n)$ and its stroke-averaged value can be written as:

$$
\begin{aligned}
& \tau_{\text {yaw }}(\hat{t})=-\rho R^{4} \bar{c}_{2}^{2}(S) \hat{r}_{c p} \Phi C_{D}\left(\alpha_{0}\right)\left|\frac{d \hat{\phi}}{d \hat{t}}\right| n \omega_{z S}, \\
& \overline{\tau_{\text {yaw }}}=-\rho R^{4} \bar{c} \hat{r}_{2}^{2}(S) \hat{r}_{c p} \Phi \overline{C_{D}(t)\left|\frac{d \widehat{\phi}}{d \hat{t}}\right|} n \omega_{z s},
\end{aligned}
$$

where $C_{D}\left(\alpha_{0}\right)$ is instantaneous drag-force coefficients as a function of (geometric) angle of attack $\alpha_{0}$ [15], $\rho$ is fluid/air density, $R$ is wing length, $\bar{c}$ is mean chord length, $\frac{d \widehat{\phi}}{d \hat{t}}$ is the non-dimensional angular velocity of the wing, $\Phi$ and $n$ are wing-flapping amplitude and frequency, $\hat{r}_{2}^{2}(S)$ is the dimensionless second moment of wing area, $\hat{r}_{c p}$ is the dimensionless center of pressure, $\omega_{z s}$ is the yaw angular velocity in the stroke plane frame, and $\phi$ is stroke angle.

During roll rotation, the wing velocity and effective angle of attack of the inner (left) wing are both altered in an opposite manner with respect to those of the outer wing (Fig. 1B). Specifically, the angular velocity around the roll axis induces a downward velocity at the left half of the stroke plane, thereby increasing the inner (left) wing's angle of attack. Similarly, it induces an upward velocity at the right half of the stroke plane and reduces the angle of attack of the outer (right) wing. Because the total aerodynamic force acting on the wing increases with the angle of attack [15], the inner wing produces a larger magnitude of force than the outer wing. Therefore similar to yaw rotation, the kinematic asymmetries lead to a net aerodynamic torque (FCT) opposite to the direction of rotation (for details, refer to roll rotation, Appendix A):

$\tau_{\text {roll }}(\hat{t}) \approx$
$-\frac{1}{2} \rho R^{4} \bar{c} \hat{r}_{2}^{2}(S) \hat{r}_{c p} \frac{\mathrm{d} C_{N}(\alpha)}{\mathrm{d} \alpha} \mid \alpha_{0}$
$\sin (\varphi) \cos ^{2}(\phi)\left|\frac{d \widehat{\phi}}{d \hat{t}}\right| \Phi n \omega_{x S}$,

at non-dimensional time $\hat{t}$, the averaged value over one wing stroke is:

$\overline{\tau_{\text {roll }}} \approx$

$-\frac{1}{2} \rho R^{4} \bar{c} \hat{r}_{2}^{2}(S) \hat{r}_{c p} \frac{\overline{\mathrm{d} C_{N}(\alpha)}}{\mathrm{d} \alpha} \mid \alpha_{0} \sin (\varphi) \cos ^{2}(\phi) \overline{\left|\frac{d \widehat{\phi}}{d \hat{t}}\right|} \Phi n \omega_{x s}$,

where $\omega_{x s}$ is the roll angular velocity in the stroke-plane frame.

During pitch rotation, wing velocity and effective angle of attack are altered asymmetrically with respect to the four quarter strokes (dorsal to middle, middle to ventral, ventral to middle, middle to dorsal: Fig. 1C). Specifically, the pitch angular velocity induces an upward velocity at the ventral (anterior) half of the stroke plane and reduces the angle of attack during the ventral to middle and the middle to ventral strokes. Similarly, it induces a downward velocity at the dorsal (posterior) half of the stroke plane and increases the angle of attack during the dorsal to middle and the middle to dorsal strokes. The collective effect of this asymmetry over one wing stroke leads to the pitch FCT (for details, refer to pitch rotation, Appendix A):

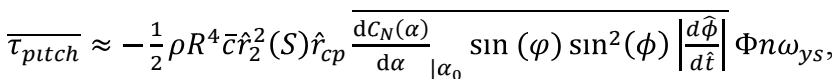

where $\omega_{y s}$ is the pitch angular velocity in the stroke-plane frame. Note that we obtained the pitch FCT by averaging the pitch torque over a wing stroke, since it is dependent on the torque difference between downstroke and upstroke therefore its instantaneous value cannot be obtained. As a result, we compare the torque production between the upstroke and downstroke to give a more intuitive description of pitch FCT (see results).

Furthermore, our model indicates that for each type of rotation, the stroke-averaged FCT acts strictly opposite to the directions of rotation (collinear with the rotation axis). In other words, there is no resultant torque about any axis perpendicular to the rotation axis (Annex A), and this is consistent with previous simulation results .

\section{Theoretical estimations of flapping counter-forces}

Similar to the aerodynamic damping (i.e., FCT) induced by body rotation, body translation also imparts an asymmetry on 
the wing kinematics, which results in a net drag opposed to the direction of translation. In accordance with the terminology of FCT, we termed the net force change as flapping counter-force (FCF). The stroke-averaged FCFs for translations along the roll $\left(x_{s}\right)$, pitch $\left(y_{s}\right)$, and yaw $\left(z_{s}\right)$ axes of the stroke plane frame (for derivations, refer to Annex B) are given by:

$$
\begin{aligned}
& \overline{\Delta F_{D-x s}}=-\rho S R \hat{r}_{1}^{1}(S) \Phi n \overline{C_{D}\left(\alpha_{0}\right) \cos ^{2}(\phi)\left|\frac{d \hat{\phi}}{d \hat{t}}\right|} v_{x S}, \\
& \overline{\Delta F_{D-y s}}=-\rho S R \hat{r}_{1}^{1}(S) \Phi n \overline{C_{D}\left(\alpha_{0}\right) \sin ^{2}(\phi)\left|\frac{d \widehat{\phi}}{d \hat{t}}\right|} v_{y s},
\end{aligned}
$$

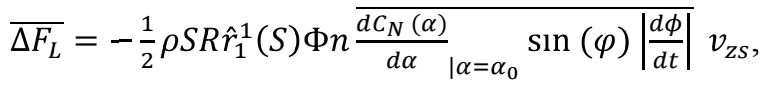

respectively, where $\hat{r}_{1}^{1}(S)$ is the non-dimensional first moment of wing area and $v_{x s}, v_{y s}$, and $v_{z s}$ represent forward/backward, lateral, and vertical velocities, respectively. Notably, in the current study we consider force coefficients $C_{D}$ and $C_{L}$ only as functions of the geometric angle of attack $\alpha_{0}$; in general, however, $C_{D}$ and $C_{L}$ are expected to be more complex functions of translational velocity and wing kinematic parameters. The effects of forward/backward translation on the aerodynamics of revolving wings have been studied by [7]. However, how lateral and vertical translations affect the aerodynamics remains unexplored. In this study, we consider only a flight near hover conditions; therefore previous quasisteady models $[15,16]$ can still apply.

\section{Stability derivatives and linearized flight dynamics during hover}

A detailed description of linearized flight dynamics can be found in [17] and [10]. Reader can also refer to [11] for related studies about linearized hovering flight dynamics. In the present context, an analytical investigation is given, based on the mathematical model of FCT and FCF. We first consider the insect body oriented with a fixed pitch angle $\chi_{0}$ (free body angle) relative to the horizontal stroke plane (Fig. 3 ). The stroke plane frame $\left(x_{\mathrm{s}}, y_{\mathrm{s}}, z_{\mathrm{s}}\right)$ is fixed relative to the body with the origin located at the wing base (Fig. 3). Here we define the body frame $\left(x_{\mathrm{b}}, y_{\mathrm{b}}, z_{\mathrm{b}}\right)$ having the same orientation with the stroke plane frame, but with the origin located at the center of gravity (Fig. 3). The distance between the wing base and the center of gravity is specified by $l_{1}$. If the insect body is modeled as a rigid body, its complete dynamics are described by the Newton-Euler equations of motion [18], which comprise six equations with translational velocity $(u, v, w)$ and angular velocity $(p, q, r)$ as unknowns. With the addition of another six kinematic equations describing the body position $(x, y, z)$ and orientation $(\phi, \theta, \psi)$ relative to the Earth-fixed frame, a complete system of equations includes twelve coupled nonlinear ordinary differential equations incorporating the aerodynamic forces $(X, Y, Z)$ and torques $(L, M, N)$, acting along or around the body axes [17]. Because body position $(x$, $y, z)$ and head angle $\psi$ do not affect the aerodynamic steady state, they can be considered separately with appropriated equations.

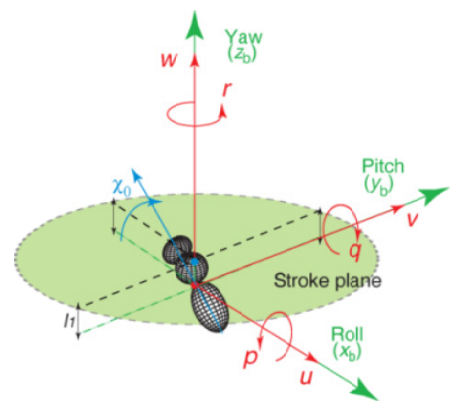

To analyze the near-hover flight dynamics, we can linearize the equations of motion using small perturbation theory. A crucial step in the linearization process is approximating the aerodynamic forces and torques as analytical functions of the perturbed motion variables (i.e., stability derivatives multiplied by the corresponding disturbance quantity of the motion variables), as well as obtaining the stability derivatives. A complete description of this method can be found in [17].

Based on the derived FCT and FCF models, we derive analytical estimations of the stability derivatives associated with flight dynamics during hover. Table 1 summarizes the nondimensional forms of stability derivatives with nonzero values. Notably, axis $x_{s}\left(y_{s}\right)$ does not coincide with axis $x_{b}\left(y_{b}\right)$ (Fig. 3); therefore a rotation around $x_{b}\left(y_{b}\right)$ corresponds to a rotation around $x_{s}\left(y_{s}\right)$ plus a linear translation of the stroke plane. We neglected the effect of this translation for simplification, which is justified in the Discussion. Other stability derivatives are expected to be zero (e.g., $X_{v}, N_{p}$ ) based on FCT and FCF models.

TABLE 1. ANALYTICAL ESTIMATIONS OF NON-

\begin{tabular}{|c|c|}
\hline $\begin{array}{l}\text { Non-dimensional } \\
\text { Stability } \\
\text { derivatives }\end{array}$ & Analytical estimations \\
\hline$X_{u}^{+}$ & $-\frac{\hat{r}_{1}^{1}(S)}{2 \hat{r}_{2}(S)} \overline{C_{D}\left(\alpha_{0}\right) \cos ^{2}(\phi)|d \hat{\phi} / d \hat{t}|}$ \\
\hline$Y_{v}^{+}$ & $-\frac{\hat{r}_{1}^{1}(S)}{2 \hat{r}_{2}(S)} \overline{C_{D}\left(\alpha_{0}\right) \sin ^{2}(\phi)|d \hat{\phi} / d \hat{t}|}$ \\
\hline$Z_{w}^{+}$ & $-\frac{\hat{r}_{1}^{1}(S)}{4 \hat{r}_{2}} \frac{\overline{d C_{N}(\alpha)}}{d \alpha \quad \mid \alpha=\alpha_{0}} \sin (\varphi)|d \hat{\phi} / d \hat{t}|$ \\
\hline$L_{p}^{+}$ & $-\frac{A \mathrm{R}}{16 \Phi} \hat{r}_{c p} \frac{\overline{\mathrm{d} C_{N}(\alpha)}}{\mathrm{d} \alpha}\left|\alpha_{0} \sin (\varphi) \cos ^{2}(\phi)\right| d \hat{\phi} / d \hat{t} \mid$ \\
\hline$L_{v}^{+}$ & $\frac{\hat{r}_{1}^{1}(S)}{4 \hat{r}_{2}(S)} A \mathrm{R} \hat{l_{1}} \hat{L} \overline{C_{D}\left(\alpha_{0}\right) \sin ^{2}(\phi)|d \hat{\phi} / d \hat{t}|}$ \\
\hline$M_{q}^{+}$ & $-\frac{A \mathrm{R}}{16 \Phi} \hat{r}_{c p} \frac{\overline{\mathrm{d} C_{N}(\alpha)}}{\mathrm{d} \alpha}\left|\alpha_{0} \sin (\varphi) \sin ^{2}(\phi)\right| d \hat{\phi} / d \hat{t} \mid$ \\
\hline$M_{u}^{+}$ & $-\frac{\hat{r}_{1}^{1}(S)}{4 \hat{r}_{2}(S)} A \mathrm{R} \hat{l}_{1} \hat{L} \overline{C_{D}\left(\alpha_{0}\right) \cos ^{2}(\phi)|d \hat{\phi} / d \hat{t}|}$ \\
\hline$N_{r}^{+}$ & $-\frac{A \mathrm{R}}{8 \Phi} \hat{r}_{c p} \overline{C_{D}(\alpha)|d \hat{\phi} / d \hat{t}|}$ \\
\hline \multicolumn{2}{|c|}{$\begin{array}{l}\text { The stability derivatives } X_{u}, Y_{v} \text { and } Z_{w} \text { are non-dimensionalized by } \rho U R \bar{c} \text {, } \\
L_{p}, M_{q} \text { and } N_{r} \text { are non-dimensionalized by } \rho U^{2} R \bar{c}^{2} / n, L_{v} \text { and } M_{u} \text { are } \\
\text { non-dimensionalized by } \rho U R \bar{c}^{2} \text {; where } U \text { equals to } 2 \Phi n R \hat{r}_{2} \text {. The superscript } \\
\text { denotes the non-dimensional values, } A \mathrm{R} \text { is aspect ratio and } \hat{L} \text { denotes the } \\
\text { body length as a fraction of wing length }(L / R) \text {. }\end{array}$} \\
\hline
\end{tabular}
DIMENSIONAL STABILITY DERIVATIVES 
Assuming hovering reference conditions:

$$
p_{e}=q_{e}=r_{e}=u_{e}=v_{e}=w_{e}=\theta_{e}=\phi_{e}=0,
$$

and the reference values of the aerodynamic forces and moments are:

$$
X_{e}=Y_{e}=L_{e}=M_{e}=N_{e}=0 \text { and } Z_{e}=\frac{g}{m} .
$$

Lastly, the linearized equations of motion in matrix form are written as:

$$
\dot{\mathbf{x}}=\left[\begin{array}{cccccccc}
\frac{X_{u}}{m} & 0 & 0 & g & 0 & 0 & 0 & 0 \\
0 & \frac{Z_{w}}{m} & 0 & 0 & 0 & 0 & 0 & 0 \\
C_{3} M_{u} & 0 & C_{3} M_{q} & 0 & 0 & 0 & 0 & 0 \\
0 & 0 & 1 & 0 & 0 & 0 & 0 & 0 \\
0 & 0 & 0 & 0 & \frac{Y_{v}}{m} & 0 & 0 & -g \\
0 & 0 & 0 & 0 & C_{4} L_{v} & C_{4} L_{p} & C_{1} N_{r} & 0 \\
0 & 0 & 0 & 0 & C_{1} L_{v} & C_{1} L_{p} & C_{2} N_{r} & 0 \\
0 & 0 & 0 & 0 & 0 & 1 & 0 & 0
\end{array}\right] \mathbf{x}=\mathbf{A x}
$$

where,

$$
\begin{aligned}
& C_{1}=\frac{I_{x z}}{I_{x x} I_{z z}-I_{x z}{ }^{2}}, C_{2}=\frac{I_{x x}}{I_{x x} I_{z z}-I_{x z}{ }^{2}}, \\
& C_{3}=\frac{1}{I_{y y}}, \quad \text { and } \quad C_{4}=\frac{I_{z z}}{I_{x x} I_{z z}-I_{x z}{ }^{2}}
\end{aligned}
$$

are constants of body moments of inertia, state vector $\mathbf{x}=(u, w, q, \theta, v, p, r, \phi)^{T} \in R^{8}, \mathbf{A}$ is the system matrix of the linearized dynamics, $m$ is body mass, $I_{x x}, I_{y y}$, and $I_{z z}$ are moments of inertia about the body axes $\left(x_{\mathrm{b}}, y_{\mathrm{b}}, z_{\mathrm{b}}\right), I_{x z}$ is the product of inertia ( $I_{y z}$ and $I_{x y}$ are zero, since the $x z$ plane is a plane of symmetry). Note that by choosing a reference body frame with the same orientation as the strokeplane frame (Fig. 3), then using the estimations of stability derivatives (in Table 1), we can decouple the hovering dynamics into longitudinal (described by $(u, w, q, \theta)^{T}$ ) and lateral (described by $\left.(v, p, r, \phi)^{T}\right)$ dynamics, as illustrated by the structure of system matrix $\mathbf{A}$.

\section{RESULTS}

\section{Robotic wing experiments on flapping counter- torques}

\section{Roll rotation}

The time courses of the measured torques around the roll, pitch, and yaw axes during roll rotation are shown in Fig. 4 ( $n$ $=0.35 \mathrm{~Hz}, \omega_{\mathrm{b}}=23 \%$ stroke, $\left.\mathrm{Re}=700\right)$. The torque production with a static stroke plane (no roll rotation) is plotted for comparison. With the roll rotation, the torques generated by the left (inner) wing (blue curves, Fig. 4) are enhanced, but the one generated by the right (outer) wing (red curves, Fig. 4) is reduced. This asymmetry leads to a continuous total roll torque (FCT) counter to the direction of turning (Fig. 4A, the time course of predicted roll torque by Eqn. 3 is also shown for comparison). The stroke-averaged value (filled green cycle, Fig. $4 \mathrm{~A}$ ) is about $6 \%$ of the peak roll torque. Also, as predicted by the FCT model (Annex A), the stroke-averaged pitch and yaw torques are both close to zero (Fig. $4 \mathrm{~B}$ and C), indicating that there is no resultant torque about any axis perpendicular to the roll axis.
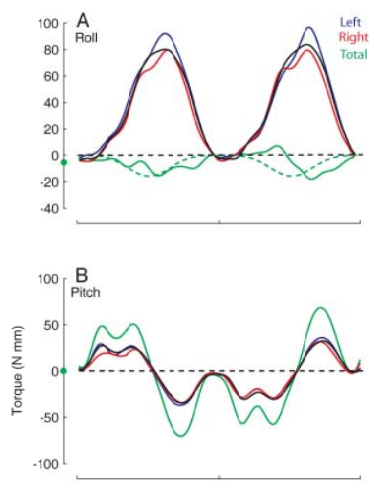

Fig. 4. Aerodynamic torque time courses during roll rotation. (A)-(C) torques about roll (A), pitch (B), and yaw (C) axes of the stroke-plane frame. The torques generated by the left and right wings are shown by blue and red curves. The total torques (right minus left) are shown by solid green curves, and the filled green cycles on the ordinates represent their averages. The predicted roll torque by the flapping counter-torque (FCT) model is shown by a dotted green curve in (A). The black curves represent the torque generation with a static stroke plane (no roll rotation).

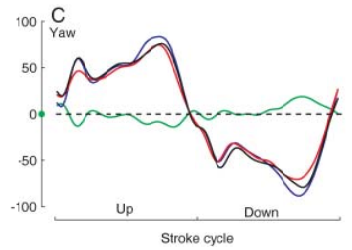

In Fig. 5, we plot the stroke-averaged roll FCT as a function of stroke frequency and angular velocity. The measured FCT has a strong linear dependence on both parameters and closely matches the theoretical estimates by Eqn. 4. Moreover, as shown in Fig. 4A, the time course of the predicted roll torque by the FCT model (dotted green) is similar to that of the measured one (solid green). Notably, the measured roll FCT has a delayed peak occurring after the mid-stroke, where the moment of arm is at maximum. Because the roll torque is associated with lift force, we expect that after the midstroke the lift force may have a value higher than the one in the mid-stroke, as observed in previous studies (Fig. 4 in [15]).

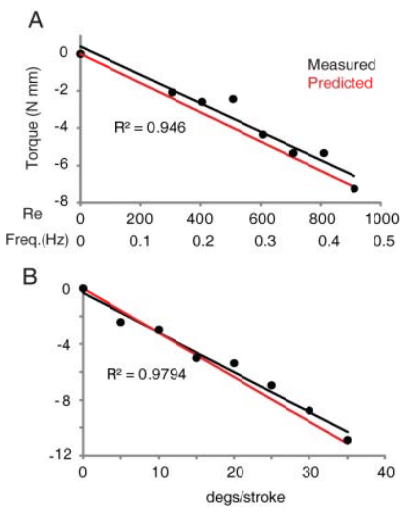

Fig. 5. Stroke-averaged roll FCT. (A) The stroke-averaged roll FCT as a function of flapping frequency is shown, and the Reynolds number associated with each frequency is also plotted. (B) The stroke-averaged roll FCT as a function of angular velocity is shown. The measured torques are shown in filled black cycles with their linear fittings (black lines). The red lines represent the predictions by Eqn. 4 .

\section{Pitch rotation}

During pitch rotation, the left and right wings have symmetric wing kinematics that ensure a symmetric torque production about the roll and yaw axes. Thus in Fig. 6, we plot 
only the time course of the measured pitch torque for a single wing and compare the torque generation during upstroke to that during downstroke $\left(n=0.35 \mathrm{~Hz}, \omega_{\mathrm{b}}=23 \%\right.$ stroke, $\left.\mathrm{Re}=700\right)$. The FCT model predicts that the pitch torque is enhanced during the first half of downstroke (dorsal to middle) and during the second half of upstroke (middle to dorsal), but it is reduced during the other two quarter strokes (ventral to middle and middle to ventral). However, the experiment results show that pitch torque is reduced only during ventral to middle stroke (first half of the blue curve, Fig. 6), and it is slightly enhanced during dorsal to middle stroke (first half of the red curve, Fig. 6). In the other two quarter strokes, there is no considerable change of pitch torque compared to those under no rotation. Nevertheless, the observed torque asymmetries at different quarter strokes still give rise to a substantial pitch FCT (solid green, Fig. 6), although its time course is different from the prediction (dotted green, Fig. 6).

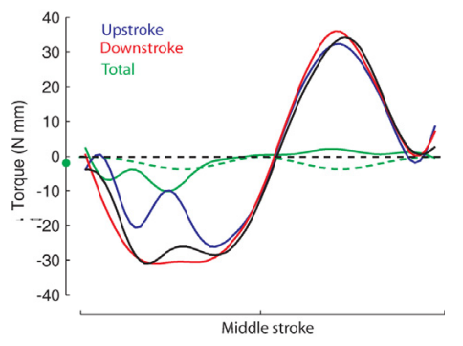

Fig. 6. Aerodynamic torque time courses about pitch axes during pitch rotation. The torques during up and down strokes are shown by blue and red curves. The total torques (red minus blue) are shown by solid green curves, and the filled green cycles on the ordinates represent their averages over one stroke. The predicted pitch torque by the FCT model is

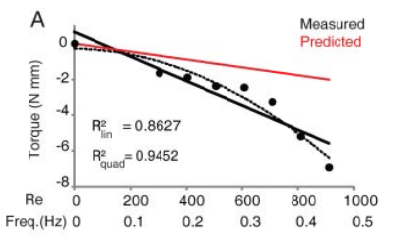

Fig. 7. Stroke-averaged pitch FCT. (A) Stroke-averaged pitch FCT as a function of flapping frequency (or Reynolds number) and (B) angular velocity. The measured torques are shown in filled black cycles with their linear (black lines) and quadratic (dotted black curve) fittings. Red lines represent the predictions by Eqn. 5 .

Fig. 7 shows the stroke-averaged pitch FCT as a function of stroke frequency and angular velocity. Although the linear dependency of FCT on angular velocity is still valid during pitch rotation (Fig. 7B), the relationship between pitch FCT and stroke frequency is better fitted by a quadratic function (Fig. 7A). The theoretical predictions, however, greatly underestimate the measured value. As will be discussed later, the failure of the prediction might result from the unmodeled aerodynamics occurring at the start of each half-stroke, where most of the variations of pitch torque are observed (Fig. 6).

\section{Yaw rotation}

During yaw rotation, the torque produced by the inner (left) wing (blue curves, Fig. 8) is enhanced during upstroke and reduced during downstroke; in contrast, the torque produced by the outer (right) wing (red curves, Fig. 8) is enhanced during downstroke and reduced during upstroke. This asymmetry leads to a continuous yaw FCT production over an entire stroke (Fig. 8C; the time course of the predicted yaw torque by Eqn. 1 is also shown for comparison). The strokeaveraged value is about $19 \%$ of the peak yaw torque (filled green cycle, Fig. 8C). Similar to roll and pitch FCT, the yaw FCT acts collinearly with the yaw axis because there is no resultant torque around the roll and pitch axes (filled green cycles, Fig. 8B and C). Unsurprisingly, the measured yaw FCT linearly increases with the stroke frequency and angular velocity (Fig. 9) and closely matches the theoretical estimates by Eqn. 2 .

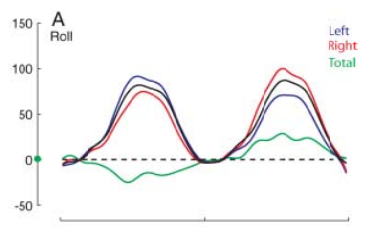

Fig. 8. Aerodynamic torque time courses during yaw rotation. (A)-(C) torques about roll (A), pitch (B), and yaw (C) axes of the stroke plane frame. Refer to the legend of Fig. 4 for details.
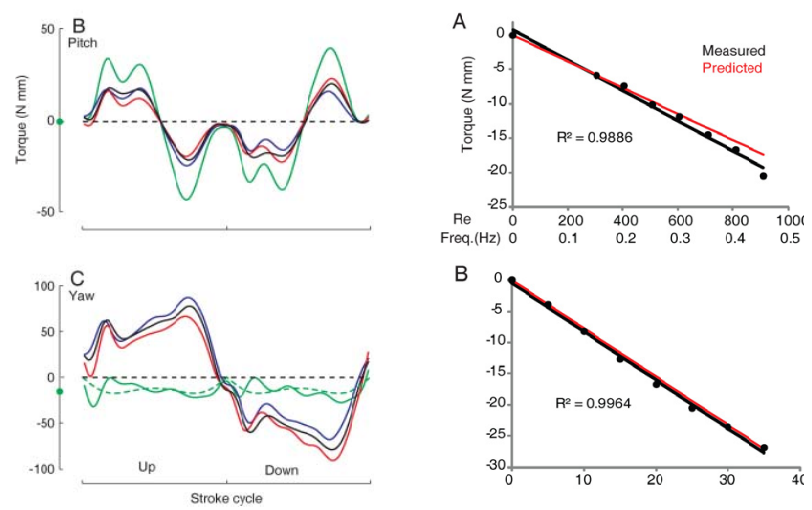

Fig. 9. Stroke-averaged yaw FCT. Refer to the legend of Fig. 5 for details. Red lines represent the predictions by Eqn. 2 .

\section{Flight stability at hovering}

Non-dimensional stability derivatives (with nonzero values) are calculated based on the analytical estimations (Table 1) and fruit fly morphological and kinematic data. The results are summarized in Table 2.

TABLE 2. NON-DIMENSIONAL STABILITY DERIVATIVES

\begin{tabular}{cccccccc}
\hline$X_{u}^{+}$ & $Y_{v}^{+}$ & $Z_{w}^{+}$ & $L_{p}^{+}$ & $L_{v}^{+}$ & $M_{q}^{+}$ & $M_{u}^{+}$ & $N_{r}^{+}$ \\
\hline-1.48 & -1.39 & -1.58 & -0.56 & 0.87 & -0.19 & -0.92 & -1.36 \\
\hline
\end{tabular}

With the stability derivatives known, the system matrix $A$ in Eqn. 11 is calculated, then the eigenvalues and corresponding eigenvectors can then be obtained accordingly. Table 3 lists the dimensional $\left(\lambda_{i}\right)$ and non-dimensional $\left(\lambda_{i}^{+}\right)$eigenvalues.

The longitudinal dynamics consist of a fast stable subsidence mode (mode 1), an unstable oscillatory mode (mode 2 ), and a slow stable subsidence mode (mode 3), which are consistent with the previous CFD results [19]. The fast subsidence mode corresponds to an in-phase coupling of $\delta u$ and $\delta q$, which results in a pitch down (up) motion accompanied by a forward (backward) translation with 
decaying magnitudes. The unstable oscillatory mode corresponds to a near out-of-phase coupling of $\delta u^{+}$and $\delta q^{+}$ (phase difference is larger than $125^{\circ}$ ); therefore, in large part of an oscillating cycle, the insects pitch up (down) while moving forward (backward), also refers to [19]). Intuitively, for the inphase coupling of initial $\delta u$ and $\delta q$, pitch FCT and the pitch torque generated by FCF $\left(\overline{\Delta F_{D-x s}} \times l_{1}\right)$ act together to reduce the angular velocity $\delta q$, resulting in stable subsequent motions. For the out-of-phase coupling of $\delta u$ and $\delta q$, however, the pitch torque induced by FCF $\left(\overline{\Delta F_{D-x S}} \times l_{1}\right)$ acts in the same direction as $\delta q$, thus magnifying the pitch angular velocity and causing the instability (see Discussion). Slow subsidence (mode 3) corresponds to a damped ascending/descending motion resulting from the FCF in a vertical direction.

TABLE 3 DIMENSIONAL AND NON-DIMENSIONAL EIGENVALUES

\begin{tabular}{|c|c|c|c|c|c|c|}
\hline & \multicolumn{3}{|c|}{ Longitudinal } & \multicolumn{3}{|c|}{ Lateral } \\
\hline & Mode 1 & Mode 2 & Mode 3 & Mode 4 & Mode 5 & Mode 6 \\
\hline$\lambda_{i}$ & -39.15 & $\begin{array}{c}14.17 \pm \\
30.62 i\end{array}$ & -5.11 & -143.02 & $\begin{array}{l}9.12 \pm \\
34.85 i\end{array}$ & -43.30 \\
\hline$\lambda_{i}^{+}$ & -0.18 & $\begin{array}{c}0.067 \pm \\
0.14 i \\
\end{array}$ & -0.024 & -0.67 & $\begin{array}{c}0.043 \pm \\
0.16 i \\
\end{array}$ & -0.20 \\
\hline
\end{tabular}

Eigenvalues (dimension of $\mathrm{Hz}$ ) are non-dimensionalized by dividing the flapping frequency $n$. The inverse of $\operatorname{Re}\left(\lambda_{i}\right)$ and $\operatorname{Re}\left(\lambda_{i}^{+}\right)$indicate the dimensional and non-dimensional time constants of the corresponding mode.

Similar to the longitudinal dynamics, the lateral dynamics consists of two subsidence modes with relative fast (mode 4) and slow (mode 6) convergences and an unstable oscillatory mode (mode 5). The fast subsidence mode corresponds to a highly damped yaw rotation (the larger value of $\lambda_{i}^{+}$means less wing-beat time to reduce the initial disturbance, Table 3) with an out-of-phase coupling of $\delta v$ and $\delta p$. The slow subsidence mode has a similar out-of-phase coupling of $\delta v$ and $\delta p$ (with difference magnitudes), but with less damped yaw rotation. The unstable oscillatory has a near in-phase coupling of $\delta v$ and $\delta p$. Intuitively, for the out-of-phase coupling of initial $\delta v$ and $\delta p$, roll FCT and the roll torque generated by FCF $\left(\overline{\Delta F_{D-y s}} \times\right.$ $l_{1}$ ) act together to reduce the angular velocity $\delta p$, resulting in stable subsequent motions. For the in-phase coupling of $\delta v$ and $\delta p$, however, the roll torque induced by FCF $\left(\overline{\Delta F_{D-y s}} \times l_{1}\right)$ acts in the same direction as $\delta p$, thus magnifying the roll angular velocity and leading to an unstable subsequent motion.

\section{DISCUSSION}

\section{Effects of unsteady aerodynamics on flapping counter-force/torque production}

In the FCT models (Eqns. 1 to 5), we considered only the translational aerodynamic force resulting from the delayed stall and ignored other aerodynamic mechanisms [20]. Previous works show that most of those unsteady aerodynamic mechanisms alter the aerodynamic force transients near stroke reversals. For example, the Kramer effect generates rotational forces before or after stroke reversals, depending on the phase of wing rotation, but wake capture occurs immediately after the stroke reversals [15].

As indicated by the plots of instantaneous torques about roll, pitch, and yaw axes (Figs. 4, 6, and 8), the peaks of roll and yaw torques occur near the middle stroke where their moments of arm are maximized. Pitch torque, however, reaches peak between the stroke reversal and the middle stroke. Therefore the unsteady aerodynamic effects, especially the wake capture, are most likely to affect pitch torque transients at the two quarter strokes after stroke reversals (ventral to middle and dorsal to middle strokes, Fig. 6). The roll and yaw torques, however, might be less affected by those unsteady effects. This might explain the discrepancy between the measured and the predicted pitch FCT (solid and dotted green, Fig. 6), especially at the two quarter strokes after the stroke reversal.

\section{Flapping counter-torque and stability derivatives during free flight}

In the current study, we assumed a constant angular velocity during rotations around each principal axis of the stroke-plane frame and measured the corresponding FCT over one wing stroke. In free flight, however, the insect is expected to experience a time-varying angular velocity on non-principal axes. Previous simulation results suggest that a rotation about a non-principal axis would also yield an FCT that linearly depends on both stroke frequency and angular velocity. However, it will be very difficult to obtain mathematical models for such rotations.

Furthermore, how FCTs depend on the accelerations is still undetermined. Although the FCT and FCF models can be used to estimate the first derivatives with respect to time (e.g., $X_{u}$ ), we are unable to estimate the second derivatives (e.g., $X_{\dot{u}}$ ). For fixed and rotary aircraft, such stability derivatives are important under some flight conditions (short-period mode, for example), in which the time constant associated is so short that the acceleration becomes critical to aerodynamics [17, 21]. However, it is still reasonable to assume that the first derivatives dominate the aerodynamics in most flight conditions $[17,21]$.

It should also be pointed out that by applying the small perturbation theory to approximate aerodynamic torques, we also neglected the nonlinear terms with higher orders, such as $Z_{u^{2}}, Z_{v^{2}}, Z_{p^{2}}, Z_{q^{2}}$, and $Z_{r^{2}}$. The estimation of these terms can be directly obtained in the FCF or FCT model. For example, it can be shown that $Z_{p^{2}}$ and $Z_{v^{2}}$ are equal to $\rho R \bar{c} \overline{\mathrm{C}_{\mathrm{L}}\left(\alpha_{0}\right) \cos ^{2}(\phi)}$ and $\rho R \bar{c} \overline{\mathrm{C}_{\mathrm{L}}\left(\alpha_{0}\right) \sin ^{2}(\phi)}$, respectively.

\section{Aerodynamic damping during non-hovering flight}

By assuming the hovering condition in our study, we consider that the effect of body velocity on flapping-wing aerodynamic model is negligible; therefore the previous quasisteady aerodynamic model [15] is applicable. However, in nonhovering flight, i.e., forward flight with high advance ratio, the 
aerodynamic model needs revision to give better estimations. Unfortunately, the understanding of the flapping-wing aerodynamics in non-hovering flight is now very limited. With respect to the forward flight, Dickson and Dickinson (2004) studied the effect of advance ratio on revolving wings and modified the quasi-steady aerodynamic model. This result can be directly applied to estimate the FCF in forward and backward translations based on Eqn. 6, with the revised lift and drag coefficients (by taking account of tip velocity ratio $\mu_{x}$ ). Similar to forward flight, vertical and lateral flights are very likely to have non-negligible effects on the aerodynamics. For example, in rotary wing aircraft a vertical descent changes the aerodynamics by reducing the downward momentum produced, and it greatly affects the lift force [17, 21]. A large descending velocity can even lead the flight into a so-called vortex-ring state, which is characterized by unstable flow conditions with erratic lift variations. Therefore an understanding of the aerodynamics, as well as the measurements of the force coefficients during non-hovering flight conditions, is desirable for further investigation of insect flight dynamics. However, as an approximation of the flight dynamics, our models provided an applicable analytical tool, which is important to designing the controllers for the flapping-wing MAVs, as well as to understanding the control strategies adapted by flying insects. For example, in the aircraft controller design, the uncertainties resulted from the neglected aerodynamics can be simply viewed as a disturbance term, which can be compensated by appropriate robust control strategies.

\section{ANNEX A}

A mathematical model of FCT for yaw rotation has been described in previous studies $[1,2]$. In this appendix, we provide a derivation of FCT models for roll and pitch rotations. For simplification, wing deviation is not considered in the current study. First, with a standard blade-element model, the lift and drag of a single flapping wing at a particular instant in the wing stroke cycle $[15,22]$ are:

$F_{\mathrm{L}}(\hat{t})=-\frac{1}{2} C_{L}(a(\hat{t})) \rho R \bar{c} \hat{r}_{2}^{2}(S) U(\hat{t})^{2}$,

$F_{\mathrm{D}}(\hat{t})=-\frac{1}{2} C_{D}(a(\hat{t})) \rho R \bar{c} \hat{r}_{2}^{2}(S) U(\hat{t})^{2}$,

where $U(\hat{t})$ is the magnitude of the wing tip velocity at nondimensional time $\hat{t}, \hat{r}_{2}(S)$ is the non-dimensional second moment of wing area, $C_{L}(a(\hat{t}))$ and $C_{D}(a(\hat{t}))$ are instantaneous lift and drag coefficients [15] as functions of effective angle of attack $a(\hat{t}), \rho$ is fluid/air density, $R$ is wing length, and $\bar{c}$ is mean chord length. Roll, pitch, and yaw torques around the center of gravity are then calculated as:

$\tau_{\text {roll }}(\hat{t})=F_{L} r_{x}=F_{L}(\hat{t}) R \hat{r}_{c p} \cos (\phi)$,

$\tau_{\text {pitch }}(\hat{t})=F_{L} r_{y}=F_{L}(\hat{t}) R \hat{r}_{c p} \sin (\phi)$,

$\tau_{\text {yaw }}(\hat{t})=F_{D} r_{z}=F_{D}(\hat{t}) R \hat{r}_{c p}$,

where $\phi$ is stroke position and $\hat{r}_{c p}$ is the normalized center of pressure on the wing. We assume that the center of pressure is located at $70 \%$ along the wingspan at any instant of time [12].
Note that roll and pitch torques are determined only by lift force $\left(F_{\mathrm{L}}\right)$, but yaw torque is determined only by drag force $\left(F_{\mathrm{D}}\right)$. Each moment of arm is determined by the orthogonal distance between the center of pressure and the corresponding principal axis $\left(r_{x}, r_{y}\right.$, and $\left.r_{z}\right)$. Notably, it can be shown that equations A3-A5 describe the torque productions regardless of the location of center of gravity, as long as we assume that it is below the wing base and along the yaw axis $z_{\mathrm{s}}$.

\section{Roll rotation}

Rotation around roll axis $x_{\mathrm{s}}$ adds a downward velocity (always normal to the stroke plane) to the left wing while increasing its effective angle of attack $\alpha$. On the contrary, however, it adds an upward velocity to the right wing and reduces its effect angle of attack. Therefore we write the lift of the left wing and the right wing as:

$F_{L-l}(\hat{t})=\frac{1}{2} C_{N}\left(\alpha_{0}+\psi\right) \sin (\varphi) \rho R \bar{c} \hat{r}_{2}^{2}(S)\left(U_{w}{ }^{2}+U_{i}{ }^{2}\right)$,

$F_{L-r}(\hat{t})=\frac{1}{2} C_{N}\left(\alpha_{0}-\psi\right) \sin (\varphi) \rho R \bar{c} \hat{r}_{2}^{2}(S)\left(U_{w}{ }^{2}+U_{i}{ }^{2}\right)$,

where $\alpha_{0}$ is the geometric angle of attack determined by wing kinematics, $\psi$ is the angle between the stroke plane and total wing velocity $U$, and $U_{w}$ and $U_{i}$ are magnitudes of wing tip velocity as a result of wing flapping and roll rotation, respectively:

$U_{w}=\left(\frac{\Phi n}{2}\right)\left|\frac{d \widehat{\phi}}{d \hat{t}}\right| R \quad$ and $U_{i}=\omega_{x s} R \cos (\phi)$,

(A8 and A9)

where $\frac{d \widehat{\phi}}{d \hat{t}}$ is the non-dimensional angular velocity of the wing, $\Phi$ and $n$ are wing-flapping amplitude and frequency, $\omega_{x s}$ is the roll angular velocity of the stroke plane, and $\phi$ is stroke position. Furthermore, $\psi$ is given by:

$$
\psi=\arctan \left(\frac{U_{i}}{U_{w}}\right) \approx \frac{2 \omega_{x s} \cos (\phi)}{\Phi n\left|\frac{d \phi}{d \bar{t}}\right|} .
$$

The magnitude of wing velocity can then be written as:

$$
U=\sqrt{\left(U_{w}{ }^{2}+U_{i}{ }^{2}\right)}=\frac{U_{w}}{\cos (\psi)}=\frac{\left(\frac{\Phi n}{2}\right)\left|\frac{d \Phi}{d t}\right| R}{\cos (\psi)} .
$$

Collectively, the roll torque of the left and right wings are calculated

$\tau_{\text {roll }-l}(\hat{t})=-\frac{1}{2} C_{N}\left(\alpha_{0}+\psi\right) \sin (\varphi) \rho R^{4} \bar{c} \hat{r}_{2}^{2}(S) \hat{r}_{c p} \cos (\phi)\left(\frac{\left(\frac{\Phi n}{2}\right)\left|\frac{d \hat{\phi}}{d t}\right|}{\cos (\psi)}\right)^{2}$, as:

$\tau_{\text {roll }-r}(\hat{t})=\frac{1}{2} C_{N}\left(\alpha_{0}-\psi\right) \sin (\varphi) \rho R^{4} \bar{c} \hat{r}_{2}^{2}(S) \hat{r}_{c p} \cos (\phi)\left(\frac{\left(\frac{\Phi n}{2}\right)\left(\frac{d \hat{\Phi}}{d t}\right)}{\cos (\psi)}\right)^{2}$.

We then approximate $C_{N}\left(a_{0} \pm \psi\right)$ as:

$C_{N}\left(\alpha_{0} \pm \psi\right) \approx C_{N}\left(\alpha_{0}\right) \pm \frac{\mathrm{d} C_{N}(\alpha)}{\mathrm{d} \alpha}{ }_{\mid \alpha_{0}} \frac{2 \omega_{x s} \cos (\phi)}{\Phi n\left|\frac{d \phi}{d t}\right|}$.

The total roll torque of a wing pair is:

$\tau_{\text {roll }}(\hat{t})=\tau_{\text {roll-l }}(\hat{t})+\tau_{\text {roll-r }}(\hat{t})$

$\approx-\frac{1}{2} \frac{\mathrm{d} c_{N}(\alpha)}{\mathrm{d} \alpha}{ }_{\mid \alpha_{0}} \sin (\varphi) \rho R^{4} \bar{c} \hat{r}_{2}^{2}(S) \hat{r}_{c p} \cos ^{2}(\phi)\left|\frac{d \hat{\Phi}}{d \hat{t}}\right| \Phi n \omega_{x S}$, 
which is the FCT for roll rotation at a non-dimensional time $\hat{t}$. The averaged value over one wing stroke is:

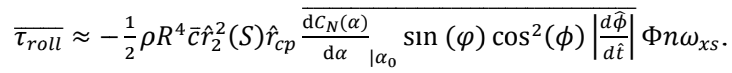

Moreover, it is easy to see that because of the geometric symmetry for lift and drag production, we have

$$
\overline{\tau_{\text {pitch }}}=\overline{\tau_{\text {yaw }}}=0
$$

\section{Pitch rotation}

Similar to roll rotation, we now derive the FCT during pitch rotation. For either left or right wing the effective angle of attack $\alpha$ is enhanced during the first half of downstroke (dorsal to middle stroke) and the second half of upstroke (middle to dorsal stroke), and it is reduced during the second half of downstroke (middle to ventral stroke) and the first half of upstroke (ventral to middle stroke). Therefore when the wing is located at the dorsal half of the stroke plane, the instantaneous lift of a single wing (i.e., left wing) can be written as:

$F_{L-l-\text { dorsal }}(\hat{t})=\frac{1}{2} C_{N}\left(\alpha_{0}+\psi\right) \sin (\varphi) \rho R \bar{c} \hat{r}_{2}^{2}(S)\left(U_{w}{ }^{2}+U_{i}{ }^{2}\right)$.

Similarily, when the wing is located at the ventral half of the stroke plane, the instantaneous lift of a single wing (i.e., left wing) is written as

$F_{L-l-v e n t r a l}(\hat{t})=\frac{1}{2} C_{N}\left(\alpha_{0}-\psi\right) \sin (\varphi) \rho R \bar{c} \hat{r}_{2}^{2}(S)\left(U_{w}{ }^{2}+U_{i}{ }^{2}\right)$.

The angle between the stroke plane and the total wing velocity $U$ is given by:

$$
\psi=\arctan \left(\frac{U_{i}}{U_{w}}\right) \approx \frac{2 \omega_{y s} \sin (\phi)}{\Phi n\left|\frac{d \widehat{\phi}}{d t}\right|},
$$

where $\omega_{y s}$ is the pitch angular velocity of the stroke plane. Therefore we have the pitch torques:

$$
\begin{aligned}
& \tau_{\text {pitch-l-dorsal }}(\hat{t})=-\frac{1}{2} C_{N}\left(\alpha_{0}+\psi\right) \sin (\varphi) \rho R^{4} \bar{c} \hat{r}_{2}^{2}(S) \hat{r}_{c p} \sin (\phi)\left(\frac{\left(\frac{\Phi \eta}{2}\right)\left|\frac{d \hat{\phi}}{d t}\right|}{\cos (\psi)}\right)^{2}, \\
& \tau_{\text {pitch-l-ventral }}(\hat{t})=\frac{1}{2} C_{N}\left(\alpha_{0}-\psi\right) \sin (\varphi) \rho R^{4} \bar{c} \hat{r}_{2}^{2}(S) \hat{r}_{c p} \sin (\phi)\left(\frac{\left(\frac{\Phi n}{2}\right)\left|\frac{d \hat{\phi}}{d \bar{t}}\right|}{\cos (\psi)}\right)^{2} .
\end{aligned}
$$

(A21 and A22)

Next we approximate $C_{N}\left(\alpha_{0} \pm \psi\right)$, as in Eqn. A14, and sum the pitch torques at two instants of time, which correspond to the symmetric stroke positions with respect to the mid-stroke:

$$
\begin{aligned}
& \tau_{\text {pitch-l-dorsal }}(\hat{t})+\tau_{\text {pitch-l-ventral }}\left(\hat{t}^{\prime}\right) \\
& =-\frac{1}{2} \frac{\mathrm{d} C_{N}(\alpha)}{\mathrm{d} \alpha} \mid \alpha_{0} \\
& \sin (\varphi) \rho R^{4} \bar{c} \hat{r}_{2}^{2}(S) \hat{r}_{c p} \sin ^{2}(\phi)\left|\frac{d \widehat{\phi}}{d \hat{t}}\right| \Phi n \omega_{y s} .
\end{aligned}
$$

We then have the averaged pitch torque of a wing pair over a complete stroke:

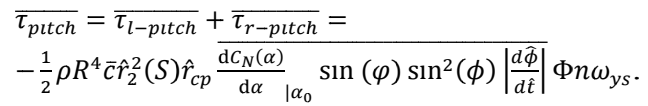

Furthermore, it is easy to see that during pitch rotation the left and right wings have symmetric wing motions; therefore symmetric torque generations around the roll and pitch axes are expected:

$$
\overline{\tau_{\text {roll }}}=\overline{\tau_{\text {yaw }}}=0 \text {. }
$$

\section{ANNEX B}

Similar to FCT mathematical models, in this Appendix we provide a derivation of FCF for body translation along the roll (forward/backward), pitch (lateral), and yaw (vertical) axes of the stroke-plane frame.
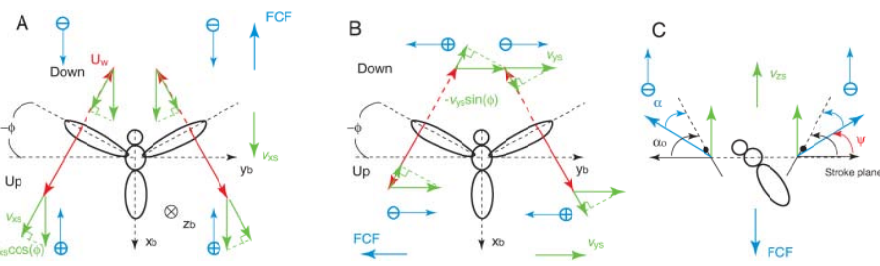

Fig. A1. Schematic view of FCF production during forward/backward (A), lateral (B), and vertical translations. Red arrows denote directions of the wing velocity (solid for upstroke, dashed for downstroke), and green arrows denote the body translational velocity. FCF (blue arrows) acts opposite to the body translation, as indicated by the enhancement (positive signs) and reduction (negative signs) of force production.

\section{Forward/backward translation}

A derivation of FCF induced by forward/back motion can be directly obtained, based on previous results from [7]. By applying blade element theory, the instantaneous drag acting on a wing can be written as:

$F_{D}(\hat{t})=\frac{1}{2} \rho S C_{D}\left(\alpha_{0}\right)\left(R \frac{d \phi}{d t}\right)^{2}\left(\hat{r}_{2}^{2}(S)+2 \hat{r}_{1}^{1}(S) \mu_{x}+\mu_{x}^{2}\right)$,

where $\mu_{x}$ is the tip velocity ratio in forward/backward translation, which is defined as:

$$
\mu_{x}=\frac{v_{x s} \cos (\phi)}{R \frac{d \phi}{d t}} .
$$

From Fig. A1A, we can see that in backward motion $\mu_{x}$ is negative during downstroke and positive during upstroke (vice versa for forward motion). Therefore,

$$
\begin{aligned}
& F_{D-u p}(\hat{t})=\frac{1}{2} \rho S C_{\mathrm{D}}\left(\alpha_{0}\right)\left(R \frac{d \phi}{d t}\right)^{2}\left(\hat{r}_{2}^{2}(S)+2 \hat{r}_{1}^{1}(S)\left|\mu_{x}\right|+\mu_{x}^{2}\right), \\
& F_{D-\text { down }}(\hat{t})=\frac{1}{2} \rho \mathrm{\rho C}_{\mathrm{D}}\left(\alpha_{0}\right)\left(R \frac{d \phi}{d t}\right)^{2}\left(\hat{r}_{2}^{2}(S)-2 \hat{r}_{1}^{1}(S)\left|\mu_{x}\right|+\mu_{x}^{2}\right) .
\end{aligned}
$$

And then we sum the drag (of a single wing) along $x_{s}$ axis at two instants of time corresponding to an identical stroke position:

$-F_{D-u p}(\hat{t}) \cos (\phi)+F_{D-\text { down }}\left(\hat{t}^{\prime}\right) \cos (\phi)$
$=-\rho R^{2} \bar{c} \hat{r}_{1}^{1}(S) \Phi n \mathrm{C}_{\mathrm{D}}\left(\alpha_{0}\right) \cos ^{2}(\phi)\left|\frac{d \hat{\phi}}{d \hat{t}}\right| v_{x s}$.

By averaging more than one wing stroke, the net drag of a wing pair is:

$\overline{\Delta F_{D-x s}}=-\rho R^{2} \bar{c} \hat{r}_{1}^{1}(S) \Phi n \overline{C_{D}\left(\alpha_{0}\right) \cos ^{2}(\phi)\left|\frac{d \hat{\phi}}{d \hat{t}}\right|} v_{x s}$.

Moreover, we can easily obtain the change of lift resulting from forward/backward motions:

$$
\overline{\Delta F_{L}}=\rho R \bar{c} \overline{\mathrm{C}_{\mathrm{L}}\left(\alpha_{0}\right) \cos ^{2}(\phi)} v_{x s}{ }^{2} .
$$


Note that the above equations also apply to the forward translation.

\section{Lateral translation}

Similar to forward translation, the instantaneous drag acting on a wing during lateral motion can be written as:

$F_{D}(\hat{t})=\frac{1}{2} \rho S_{\mathrm{D}}\left(\alpha_{0}\right)\left(R \frac{d \phi}{d t}\right)^{2}\left(\hat{r}_{2}^{2}(S)+2 \hat{r}_{1}^{1}(S) \mu_{y}+\mu_{y}{ }^{2}\right)$,

where $\mu_{y}$ is the tip velocity ratio in lateral translation, which is defined as:

$$
\mu_{y}=(-1)^{i} \frac{v_{y s} \sin (\phi)}{R \frac{d \phi}{d t}},
$$

where $i=0$ and 1 for the left and right wings, respectively. We can see in Fig. A1B that during a lateral motion to the right (left), the net drag of a wing pair along the pitch axis $y_{s}$ is always pointing to the left (right) at any instant of time (i.e., an enhanced drag to the left (positive $\mu_{y}$ ) and decreased drag to the right (negative $\mu_{y}$ ) during downstroke at ventral half of the stroke plane, Fig. A1B). Collectively, we have

$\Delta F_{D-y s}(\hat{t})=-\rho R^{2} \bar{c} \hat{r}_{1}^{1}(S) \Phi n C_{D}\left(\alpha_{0}\right) \sin ^{2}(\phi)\left|\frac{d \hat{\phi}}{d \hat{t}}\right| v_{y s}$,

and the stroke averaged value is:

$\overline{\Delta F_{D-y s}}=-\rho R^{2} \bar{c} \hat{r}_{1}^{1}(S) \Phi n \overline{C_{D}\left(\alpha_{0}\right) \sin ^{2}(\phi)\left|\frac{d \hat{\phi}}{d \hat{t}}\right|} v_{y s}$.

Moreover, the change of lift because of lateral motions is:

$\overline{\Delta F_{L}}=\rho R \bar{c} \overline{\mathrm{C}_{\mathrm{L}}\left(\alpha_{0}\right) \sin ^{2}(\phi)} v_{y s}{ }^{2}$.

\section{Vertical translation}

A vertical translation changes the effective angle of attack at a wing section by:

$$
\psi=\arctan \left(\frac{v_{z s}}{r \mid \frac{d \phi}{d t}}\right),
$$

where $\psi$ is the angle between total wing velocity, and the stroke plane $r$ is the spanwise location of the wing section. The magnitude of total wing velocity is:

$$
U=\frac{r\left|\frac{d \phi}{d t}\right|}{\cos (\psi)}=\sqrt{\left(r \frac{d \phi}{d t}\right)^{2}+v_{z s}^{2}} .
$$

Thus the sectional lift force during ascent is given by:

$\mathrm{d} F_{L}(\hat{t})=\frac{1}{2} \rho(d r) c(r) C_{N}\left(\alpha_{0}-\psi\right) \sin (\varphi)\left(\frac{r\left|\frac{d \phi}{d t}\right|}{\cos (\psi)}\right)^{2}$.

Furthermore, the change of the sectional lift force resulting from vertical velocity is:

$$
\begin{gathered}
\Delta \mathrm{d} F_{L}(\hat{t})=\frac{1}{2} \rho(d r) c(r)\left(r\left|\frac{d \phi}{d t}\right|\right)^{2}\left[C_{N}\left(\alpha_{0}-\psi\right)\left(\frac{1}{\cos (\psi)}\right)^{2}-C_{N}\left(\alpha_{0}\right)\right] \sin (\varphi) \\
\approx-\frac{1}{2} \rho(d r) c(r) r\left|\frac{d \phi}{d t}\right| \sin (\varphi)\left[\frac{d C_{N}(\alpha)}{d \alpha}{ }_{\mid \alpha=\alpha_{0}} v_{z s}\right] .
\end{gathered}
$$

Integrate the above equation over an entire wingspan, and we have the net lift change of a wing pair:

$\Delta F_{L}(\hat{t})=-\frac{1}{2} \rho R^{2} \bar{c} \Phi n\left|\frac{d \hat{\phi}}{d \hat{t}}\right| \frac{d C_{N}(\alpha)}{d \alpha}{ }_{\alpha=\alpha_{0}} \sin (\varphi) \hat{r}_{1}^{1}(S) v_{z S}$.
The stroke averaged value is:

$\overline{\Delta F_{L}}=-\frac{1}{2} \rho R^{2} \bar{c} \hat{r}_{1}^{1}(S) \Phi n{\overline{\frac{d C_{N}(\alpha)}{d \alpha}} \mid \alpha=\alpha_{0}}_{\sin (\varphi)\left|\frac{d \widehat{\phi}}{d \hat{t}}\right|} v_{z S}$.

\section{ACKNOWLEDGMENTS}

This work was supported in part by NSF Grant\#0545931.

\section{REFERENCES}

[1] B. Cheng, S. Fry, Q. Huang, and X. Deng, "Aerodynamic damping during rapid flight maneuvers in the fruit fly Drosophila " Journal of Experimental Biology, vol. 213, pp. 602-612, 2009.

[2] T. L. Hedrick, B. Cheng, and X. Deng, "Wingbeat Time and the Scaling of Passive Rotational Damping in Flapping Flight," Science, vol. 324, pp. 252-255, April 10, 2009.

[3] S. N. Fry, R. Sayaman, and M. H. Dickinson, "The aerodynamics of freeflight maneuvers in Drosophila," Science, vol. 300, pp. 495-498, Apr 2003.

[4] C. P. Ellington, "The Aerodynamics of Hovering Insect Flight .6. Lift and Power Requirements," Philosophical Transactions of the Royal Society of London Series B-Biological Sciences, vol. 305, pp. 145-181, 1984.

[5] A. R. Ennos, "The Kinematics and Aerodynamics of the Free Flight of Some Diptera," Journal of Experimental Biology, vol. 142, pp. 49-85, Mar 1989.

[6] C. T. David, "The relationship between body angle and flight speed in freeflying Drosophila," Physiol. Entomol, vol. 3, pp. 191-195, 1978.

[7] W. B. Dickson and M. H. Dickinson, "The effect of advance ratio on the aerodynamics of revolving wings," Journal of Experiment Biology, vol. 207, pp. 4269-4281, November 152004.

[8] H. Buelthoff, T. Poggio, and C. Wehrhahn, "3-D Analysis of the Flight Trajectories of Flies (Drosophila rnelanogaster)," Z. Naturforsch, vol. 35c, pp. 811-815, 1980 .

[9] M. Sun and J. K. Wang, "Flight stabilization control of a hovering model insect," Journal of Experimental Biology, vol. 210, pp. 2714-2722, Aug 2007.

[10] G. K. Taylor and A. L. R. Thomas, "Dynamic flight stability in the desert locust Schistocerca gregaria," Journal of Experimental Biology, vol. 206, pp. 2803-2829, Aug 2003.

[11] X. Y. Deng, L. Schenato, W. C. Wu, and S. S. Sastry, "Flapping flight for biomimetic robotic insects: Part I - System modeling," IEEE Transactions on Robotics, vol. 22, pp. 776-788, Aug 2006.

[12] S. N. Fry, R. Sayaman, and M. H. Dickinson, "The aerodynamics of hovering flight in Drosophila," Journal of Experimental Biology, vol. 208, pp. 2303-2318, Jun 2005.

[13]J. H. Marden, "Maximum Lift Production During Takeoff in Flying Animals," Journal of Experiment Biology, vol. 130, pp. 235-258, July 1, 1987.

[14]R. Dudley, The biomechanics of insect flight: Princeton University Press, 2000.

[15]M. H. Dickinson, F. O. Lehmann, and S. P. Sane, "Wing rotation and the aerodynamic basis of insect flight," Science, vol. 284, pp. 1954-1960, Jun 1999.

[16] S. P. Sane and M. H. Dickinson, "The aerodynamic effects of wing rotation and a revised quasi-steady model of flapping flight," Journal of Experimental Biology, vol. 205, pp. 1087-1096, Apr 2002.

[17]B. Etkin and L. D. Reid, Dynamics of Flight: Stability and Control. New York: Wiley, 1996.

[18]R. M. Murray, Z. Li, and S. S. Sastry, A Mathematical Introduction to Robotic Manipulation: CRC, 1994.

[19]M. Sun and Y. Xiong, "Dynamic flight stability of a hovering bumblebee," Journal of Experimental Biology, vol. 208, pp. 447-459, Feb 2005.

[20] S. P. Sane, "The aerodynamics of insect flight," Journal of Experimental Biology, vol. 206, pp. 4191-4208, December 12003.

[21]R. W. Prouty, Helicopter Performance, Stability, and Control. Boston: PWS Engineering, 1986.

[22] S. P. Sane and M. H. Dickinson, "The control of flight force by a flapping wing: lift and drag production," Journal of Experimental Biology, vol. 204, pp. 2607-2626, 2001. 